\title{
Numerical solution of fractional boundary value problem with caputo-fabrizio and its fractional integral
}

\author{
M. Moumen Bekkouche ${ }^{1}$ (D) I. Mansouri ${ }^{1}$ - A. A. Azeb Ahmed ${ }^{1}$
}

Received: 20 October 2021 / Revised: 4 January 2022 / Accepted: 24 January 2022 /

Published online: 4 February 2022

(c) The Author(s) under exclusive licence to Korean Society for Informatics and Computational Applied Mathematics 2022

\begin{abstract}
In this article, we investigate the existence and uniqueness of the solution of a fractional boundary value problem with conformable fractional derivation of the Caputo-Fabrizio type. In order to study this problem we used a new definition of fractional integral as an inverse of the conformable fractional derivative of Caputo-Fabrizio, therefore, so we transformed the problem to a equivalent linear Volterra-Fredholm integral equations of the second kind, and taking sufficient conditions existence and uniqueness of this solution is proven based on the results obtained. The analytical study is followed by a complete numerical study.
\end{abstract}

Keywords Caputo-Fabrizio fractional derivative $\cdot$ Fractional boundary value problem · Fractional integral · Volterra-Fredholm integral equation

Mathematics Subject Classification 34K37 · 26A33 · 34A08 · 45B05

\section{Introduction}

In recent years, the mathematical models that involving fractional order derivative have become dominant in many fields due to their remarkable importance as being more accurate than the classical order models [14, 24, 25]. In order to advancement of fractional calculus, many researchers have focused to investigate the solution $\mathrm{s}$ of nonlinear differential equations involves several fractional differential operators

M. Moumen Bekkouche
moumen-med@univ-eloued.dz
I. Mansouri
mansouri-ikram@univ-eloued.dz

A. A. Azeb Ahmed

azizazeb@gmail.com

1 Department of Mathematics, Faculty of Exact Sciences, El Oued University, 39000 El Oued, Algeria 
like: Riemann-Lioville, Hilfer, and Caputo ... ect (see [3, 26, 28]). However, these operators have a power law kernel and have limitations in modeling physical problems. To overcome this difficulty in 2015, a new fractional derivation approach known as Caputo and Fabrizio (C-F) [8] was developed, characterized by without singular kernel, which gave it importance in modeling a certain class of real-world problem, which follow the exponential decay law.

In recent times, several authors contributed to the development and simulation of CFFDE, to know some applications presented for example, the fractional C-F derivative has been employed for the description of many complex biological systems, including the rabies model [4], the Cancer treatment by radiotherapy model [9], the mathematical model for the transmission of Covid-19 [6], and as well as a new model of human liver [5], as it has been used in many other fields, such as further extend the groundwater flow model [1], . . . ect. To know more characteristics and application (see [2, 7, 11-13, $16,31])$.

In this paper, We study the existence and uniqueness of the solution of the fractional differential equation boundary value problem with the Caputo-Fabrizio, as follows:

$$
\left\{\begin{array}{l}
\mathscr{D}^{(\rho)} v(t)+q(t) v(t)=f(t), \quad 0 \leq t \leq 1 \\
v(0)=a, \quad v(1)=b
\end{array}\right.
$$

where $1<\rho<2$ is a real number, $q$ is the potential function, and $f:[0,1] \rightarrow \mathbb{R}$ is continuous. and $\mathscr{D}^{(\rho)}$ is the new fractional derivative, and we introduce a new definition of its fractional integral with some properties, using this fractional integral upon problem (1) to obtain an equivalent linear Volterra-Fredholm integral equations of second kind. Finally, by the means of some theorems, the existence and uniqueness of solutions are obtained, and we introduce an algorithm for finding a numerical solution of this problem class.

\section{Preliminaries}

For the convenience of the reader, we present here the necessary definitions and lemmas from fractional calculus theory. These definitions can be found in the recent literature.

Definition 1 Let $\Omega=[a, b]$ be a finite interval on the real axis $\mathbb{R}$. The left-sided Caputo-Fabrizio fractional derivative ${ }^{C F} \mathscr{D}_{\alpha^{+}}^{\gamma} v$ of order $\gamma \in[0,1[$ of a function $v$ is defined as follows:

$$
\mathscr{D}^{(\gamma)} v(t)=\frac{M(\gamma)}{1-\gamma} \int_{a}^{t} v^{\prime}(\tau) \exp \left[-\frac{\gamma(t-\tau)}{1-\gamma}\right] \mathrm{d} \tau
$$

where $\gamma \in[0,1]$ and $a \in]-\infty, t), v \in H^{1}(a, b), b>a$, and $M(\gamma)$ is a normalization function such that $M(0)=M(1)=1$. 
Definition 2 Let $n \geq 1$, and $\gamma \in[0,1]$ the fractional derivative $\mathscr{D}^{(\gamma+n)} f$ of order $(n+\gamma)$ is defined by

$$
\mathscr{D}^{(\gamma+n)} v(t):=\mathscr{D}_{t}^{(\gamma)}\left(\mathscr{D}^{(n)} v(t)\right)=\frac{M(\gamma)}{1-\gamma} \int_{\alpha}^{t} v^{(n+1)}(\tau) \exp \left[-\frac{\gamma(t-\tau)}{1-\gamma}\right] \mathrm{d} \tau
$$

Such that

$$
\mathscr{D}^{(\gamma+n)} v(t)=\frac{M(\gamma)}{1-\gamma} \int_{\alpha}^{t} v^{(n+1)}(\tau) \exp \left[-\frac{\gamma(t-\tau)}{1-\gamma}\right] \mathrm{d} \tau
$$

Definition 3 (A new fractional integral) [21] Let $n \geq 1, \gamma \in[0,1]$, and $v \in \mathcal{C}[\alpha, \beta]$. The formula :

$$
I_{\alpha}^{n+\gamma} v(t)=\frac{1}{M(\gamma) \cdot n !} \int_{\alpha}^{t}(t-\tau)^{n-1}[\gamma(t-\tau)+n(1-\gamma)] v(\tau) \mathrm{d} \tau
$$

where $M(\gamma)$, is a normalization function such that $M(0)=M(1)=1$ is a new fractional integral of order $(n+\gamma)$, and it's as an inverse of the conformable fractional derivative of Caputo of order $(n+\gamma)$.

Lemma 1 ([21]) Let $\rho \in(n, n+1), n=[\rho] \geq 0$. Assume that $v \in \mathcal{C}^{n}[\alpha, \beta]$, then those statements hold:

1. if $v(\alpha)=0$, then $\mathscr{D}^{(\rho)}\left(I_{\alpha}^{\rho} v(t)\right)=v(t)$.

2. $I_{\alpha}^{\rho}\left(\mathscr{D}^{(\rho)} v(t)\right)=v(t)+\sum_{i=0}^{n} \alpha_{i} t^{i}, \alpha_{i} \in \mathbb{R} i=0,1, \ldots, n$.

\section{Analytic study}

In the following, we suppose the function $M(\gamma)=1$.

Lemma 2 Given $q \in \mathcal{C}[0,1]$, and $1<\rho<2$, the solution of

$$
\begin{aligned}
& \mathscr{D}^{(\rho)} v(t)+q(t) v(t)=f(t), \quad 0 \leq t \leq 1 \\
& v(0)=a, \quad v(1)=b
\end{aligned}
$$

is solution of linear integral equation Volterra-Fredholm of the second kind

$$
v(t)+\int_{0}^{t} L(t, \tau) v(\tau) \mathrm{d} \tau+\int_{0}^{1} F(t, \tau) v(\tau) \mathrm{d} \tau=g(t)
$$

where $g(t)=(b-a) t+a+\int_{0}^{t}[\gamma(t-\tau)+(1-\gamma)] f(\tau) \mathrm{d} \tau+\int_{0}^{1} t(\gamma \tau-1) f(\tau) \mathrm{d} \tau$,

$$
L(t, \tau)=[\gamma(t-\tau)+(1-\gamma)] q(\tau) \text { and } F(t, \tau)=t(\gamma \tau-1) q(\tau) .
$$


Proof We may apply Lemma 1 to Convert Eq. (4) to an equivalent integral equation

$$
\begin{aligned}
& I_{0}^{\rho}\left(\mathscr{D}^{(\rho)} v(t)\right)=I_{0}^{\rho}(f(t)-q(t) v(t)) \\
& \quad \Rightarrow v(t)+c t+d=\int_{0}^{t}[\gamma(t-\tau)+(1-\gamma)](f(\tau)-q(\tau) v(\tau)) \mathrm{d} \tau
\end{aligned}
$$

Using boundary conditions $v(0)=a, v(1)=b$, we obtain $d=-v(0)=-a$, and

$$
c=a-b+\int_{0}^{1}(1-\gamma \tau)(f(\tau)-q(\tau) v(\tau)) \mathrm{d} \tau .
$$

Hence, the unique solution of problem (4) is

$$
\begin{aligned}
v(t)= & (b-a) t+a+\int_{0}^{t}[\gamma(t-\tau)+(1-\gamma)](f(\tau)-q(\tau) v(\tau)) \mathrm{d} \tau \\
& +\int_{0}^{1} t(\gamma \tau-1)(f(\tau)-q(\tau) v(\tau)) \mathrm{d} \tau \\
= & (b-a) t+a+\int_{0}^{t}[\gamma(t-\tau)+(1-\gamma)] f(\tau) \mathrm{d} \tau+\int_{0}^{1} t(\gamma \tau-1) f(\tau) \mathrm{d} \tau \\
& -\int_{0}^{t}[\gamma(t-\tau)+(1-\gamma)] q(\tau) v(\tau) \mathrm{d} \tau-\int_{0}^{1} t(\gamma \tau-1) q(\tau) v(\tau) \mathrm{d} \tau \\
= & g(t)-\int_{0}^{t} L(t, \tau) v(\tau) \mathrm{d} \tau-\int_{0}^{1} F(t, \tau) v(\tau) \mathrm{d} \tau .
\end{aligned}
$$

The proof is complete.

\section{Existence and uniqueness of the solution}

The classical approach to proving the existence and uniqueness of the solution of (5) is the Picard method. This consists of the simple iteration for $n=1,2, \ldots$

$$
v_{n}(t)=g(t)+\int_{0}^{t} L(t, \tau) v_{n-1}(\tau) \mathrm{d} \tau+\int_{0}^{1} F(t, \tau) v_{n-1}(\tau) \mathrm{d} \tau
$$

with $v_{0}(t)=g(t)$. For ease of manipulation, it is convenient to introduce

$$
u_{n}(t)=v_{n}(t)-v_{n-1}(t), \quad n=1,2, \ldots
$$

with $u_{0}(t)=g(t)$. On subtracting from (6), the same equation with $n$ replaced by $n-1$, an we see that

$$
u_{n}(t)=\int_{0}^{t} L(t, \tau) u_{n-1}(\tau) \mathrm{d} \tau+\int_{0}^{1} F(t, \tau) u_{n-1}(\tau) d \tau, \quad n=1,2, \ldots
$$


Also, from (7)

$$
v_{n}(t)=\sum_{i=0}^{n} u_{i}(t)
$$

The following theorem uses this iteration to prove the existence and uniqueness of the solution under quite restrictive conditions, namely that $L(x, \tau), F(t, \tau)$ and $g(t)$ are continuous.

Theorem 1 If $g(t)$ is continuous in $0 \leq t \leq 1$, and the function $F(t, \tau), L(t, \tau)$ are continuous in $0 \leq \tau \leq t \leq 1$, and $\max _{0 \leq \tau \leq t \leq 1}|F(t, \tau)|<1$, then the integral Eq. (5) possesses a unique continuous solution for $0 \leq t \leq 1$.

Proof Choose $\lambda_{1}, \lambda_{2}$ and $\lambda_{3}$ such that

$$
\begin{aligned}
|g(t)| & \leq \lambda_{1}, 0 \leq t \leq 1 \\
|L(t, \tau)| & \leq \lambda_{2}, 0 \leq \tau \leq t \leq 1 \\
|F(t, \tau)| & \leq \lambda_{3}, 0 \leq \tau \leq t \leq 1 \text { where } \lambda_{3}<1 .
\end{aligned}
$$

We first prove by induction that

$$
\left|u_{n}(t)\right| \leq \frac{\lambda_{1}\left(\lambda_{2} t\right)^{n}}{n !}+\lambda_{1} \lambda_{3}^{n}, \quad 0 \leq t \leq 1, \quad n=0,1, \ldots
$$

If we assume that (10) is true for $n-1$, then from (8)

$$
\begin{aligned}
\left|u_{n}(t)\right| & \leq \frac{\lambda_{1} \lambda_{2}^{n}}{(n-1) !} \int_{0}^{t} \tau^{n-1} \mathrm{~d} \tau+\lambda_{1} \lambda_{3}^{n} \\
& =\frac{\lambda_{1}\left(\lambda_{2} t\right)^{n}}{n !}+\lambda_{1} \lambda_{3}^{n} .
\end{aligned}
$$

Since (10) is obviously true for $n=0$, it holds for all $n$. This bound makes it obvious that the sequence $v_{n}(t)$ in (9) converges, and we can write

$$
v(t)=\sum_{i=0}^{\infty} u_{i}(t)
$$

We now show that this $v(t)$ satisfies Eq. (5). The series (11) is uniformly convergent since the terms $u_{i}(t)$ are dominated by $\lambda_{1}\left(\lambda_{2} t\right)^{i} / i !+\lambda_{1} \lambda_{3}^{i}$. Consequently, we can interchange the order of integration and summation in the following expression to obtain 


$$
\begin{aligned}
\int_{0}^{t} L(t, \tau) \sum_{i=0}^{\infty} u_{i}(\tau) \mathrm{d} \tau+\int_{0}^{1} F(t, \tau) \sum_{i=0}^{\infty} u_{i}(\tau) \mathrm{d} \tau & =\sum_{i=0}^{\infty} \int_{0}^{t} L(t, \tau) u_{i}(\tau) \mathrm{d} \tau \\
& +\sum_{i=0}^{\infty} \int_{0}^{1} F(t, \tau) u_{i}(\tau) \mathrm{d} \tau \\
& =\sum_{i=0}^{\infty} u_{i+1}(\tau) \\
& =\sum_{i=0}^{\infty} u_{i}(\tau)-g(t)
\end{aligned}
$$

Each of the $u_{i}(t)$ is clearly continuous. Therefore $v(t)$ is continuous, since it is the limit of a uniformly convergent sequence of continuous functions.

To show that $v(t)$ is the only continuous solution, suppose there exists another continuous solution $\tilde{v}(t)$ of (5) Then

$$
v(t)-\tilde{v}(t)=\int_{0}^{t} L(t, \tau)(v(\tau)-\tilde{v}(\tau)) \mathrm{d} \tau+\int_{0}^{1} F(t, \tau)(v(\tau)-\tilde{v}(\tau)) \mathrm{d} \tau
$$

since $f(t)$ and $\tilde{f}(t)$ are both continuous, there exists a constant $C$ such that

$$
|v(t)-\tilde{v}(t)| \leq C, \quad 0 \leq t \leq 1
$$

Substituting this into (12)

$$
|v(t)-\tilde{v}(t)| \leq C\left(\lambda_{2} t+\lambda_{3}\right), \quad 0 \leq t \leq 1
$$

and repeating the step shows that

$$
|v(t)-\tilde{v}(t)| \leq C\left(\frac{\left(\lambda_{2} t\right)^{n}}{n !}+\lambda_{3}^{n}\right), \quad 0 \leq t \leq 1, \text { for any } n
$$

For a large enough $n$, the right-hand side is arbitrarily small, therefore, we must have

$$
v(t)=\tilde{v}(t), \quad 0 \leq t \leq 1
$$

Theorem 2 If $f(t), q(t)$ are continuous in $[0,1]$, and $\max _{0 \leq t \leq 1}|q(t)|<1$, then the fractional boundary value problem (1) possesses a unique continuous solution for $0 \leq t \leq 1$

Proof If $f(t), q(t)$ are continuous in $[0,1]$, then it is clear that the following functions

$$
g(t)=(b-a) t+a+\int_{0}^{t}[\gamma(t-\tau)+(1-\gamma)] f(\tau) \mathrm{d} \tau+\int_{0}^{1} t(\gamma \tau-1) f(\tau) \mathrm{d} \tau,
$$




$$
\begin{aligned}
& L(t, \tau)=[\gamma(t-\tau)+(1-\gamma)] q(\tau), \\
& F(t, \tau)=t(\gamma \tau-1) q(\tau),
\end{aligned}
$$

are continuous, and $|F(t, \tau)|=|t(\gamma \tau-1) q(\tau)| \leq|q(\tau)|<1, \forall t, \tau \in[0,1]$, which means that integral Eq. (5) possesses a unique continuous solution for $0 \leq t \leq 1$. Therefore, there is a unique continuous solution of the fractional boundary value problem (1) for $0 \leq t \leq 1$.

\section{Numerical study}

In this section, we introduce an algorithm for finding a numerical solution of linear Volterra-Fredholm integral equations of the second kind, the methods based upon trapezoidal rule. For all $N \in \mathbb{N}$, Here the interval $[0,1]$ in to $N$ equal sub-intervals, where $h=(\beta-\alpha) / N$, and $t_{i}=\alpha+i \cdot h$ for all $i \in\{0 \cdots N\}$.

The formula of the numerical integration is:

$$
\int_{\alpha}^{\beta} f(\tau) \mathrm{d} \tau \approx \frac{h}{2}\left[f(\alpha)+2 \sum_{j=1}^{N} f\left(t_{j}\right)+f(\beta)\right]
$$

we apply this formula in Eq. (5), and we obtain:

$$
\begin{aligned}
g\left(t_{i}\right)= & v\left(t_{i}\right)+\frac{h}{2}\left[L\left(t_{i}, t_{0}\right) v\left(t_{0}\right)+2 \sum_{j=1}^{i-1} L\left(t_{i}, t_{j}\right) v\left(t_{j}\right)+L\left(t_{i}, t_{i}\right) v\left(t_{i}\right)\right] \\
& +\frac{h}{2}\left[F\left(t_{i}, t_{0}\right) v\left(t_{0}\right)+2 \sum_{j=1}^{N-1} F\left(t_{i}, t_{j}\right) v\left(t_{j}\right)+F\left(t_{i}, t_{N}\right) v\left(t_{N}\right)\right] \\
& \Rightarrow \forall i=0, \ldots, N, g_{i}=v_{i}+\frac{h}{2}\left[L_{i 0} v_{0}+2 \sum_{j=1}^{i-1} L_{i j} v_{j}+L_{i i} v_{i}\right] \\
& +\frac{h}{2}\left[F_{i 0} v_{0}+2 \sum_{j=1}^{N-1} F_{i j} v_{j}+F_{i N} v_{N}\right]
\end{aligned}
$$

This leads to

$$
\begin{aligned}
& \frac{h}{2}\left(L_{i 0}+F_{i 0}\right) v_{0}+h \sum_{j=1}^{i-1}\left(L_{i j}+F_{i j}\right) v_{j}+\frac{h}{2}\left(\frac{2}{h}+L_{i i}+2 F_{i i}\right) v_{i} \\
& \quad+h \sum_{j=i+1}^{N-1} F_{i j} v_{j}+\frac{h}{2} F_{i N} v_{N}=g_{i}
\end{aligned}
$$


So

$$
\begin{aligned}
& h \sum_{j=1}^{i-1}\left(L_{i j}+F_{i j}\right) v_{j}+\frac{h}{2}\left(\frac{2}{h}+L_{i i}+2 F_{i i}\right) v_{i}+h \sum_{j=i+1}^{N-1} F_{i j} v_{j} \\
& \quad=g_{i}-\frac{h}{2}\left(L_{i 0}+F_{i 0}\right) v_{0}-\frac{h}{2} F_{i N} v_{N}
\end{aligned}
$$

Finally, we get a system of $N-1$ equations, which is:

$$
A V=B
$$

when $V=\left(v_{1}, \ldots, v_{N-1}\right), A=\left(a_{i j}\right)_{i, j=1, \ldots, N-1}$ and $B=\left(b_{i}\right)_{i=1, \ldots, N-1}$;

$$
a_{i j}= \begin{cases}h \cdot\left(L_{i i}+2 F_{i i}\right) / 2+1 & \text { if } j=i \\ h \cdot\left(L_{i j}+F_{i j}\right) & \text { if } j=1: i-1 \\ h \cdot F_{i j} & \text { if } j=i+1: N-1\end{cases}
$$

and

$$
b_{i}=g_{i}-\frac{h}{2}\left(L_{i 0}+F_{i 0}\right) a-\frac{h}{2} F_{i N} b \quad \text { if } i=1, \ldots, N-1
$$

\section{Numerical result}

In this part, we give three numerical examples to illustrate the above methods to solve the Volterra-Fredholm linear integral equations of the second kind. The exact solution is known and used to show that the numerical solution obtained with our methods is correct. We used MATLAB to solve these examples.

Example 1 Consider the following fractional boundary value problem:

$$
\begin{aligned}
& \mathscr{D}^{(\rho)} v(t)+q(t) v(t)=f(t), \quad 0 \leq t \leq 1 \\
& v(0)=1, \quad v(1)=\frac{e^{-1}+2}{2}
\end{aligned}
$$

where $\rho=1.5, q(t)=1-\frac{t^{4}}{20}, \quad f(t)=\frac{t}{4}\left(3 e^{-t}-t-1\right)$, with the exact solution $v(t)=\frac{e^{-t}+t+1}{2}$.

Example 2 Consider the following fractional boundary value problem:

$$
\begin{aligned}
& \mathscr{D}^{(\rho)} v(t)+q(t) v(t)=f(t), \quad 0 \leq t \leq 1 \\
& v(0)=1, \quad v(1)=\frac{e^{-1}+1}{2}
\end{aligned}
$$



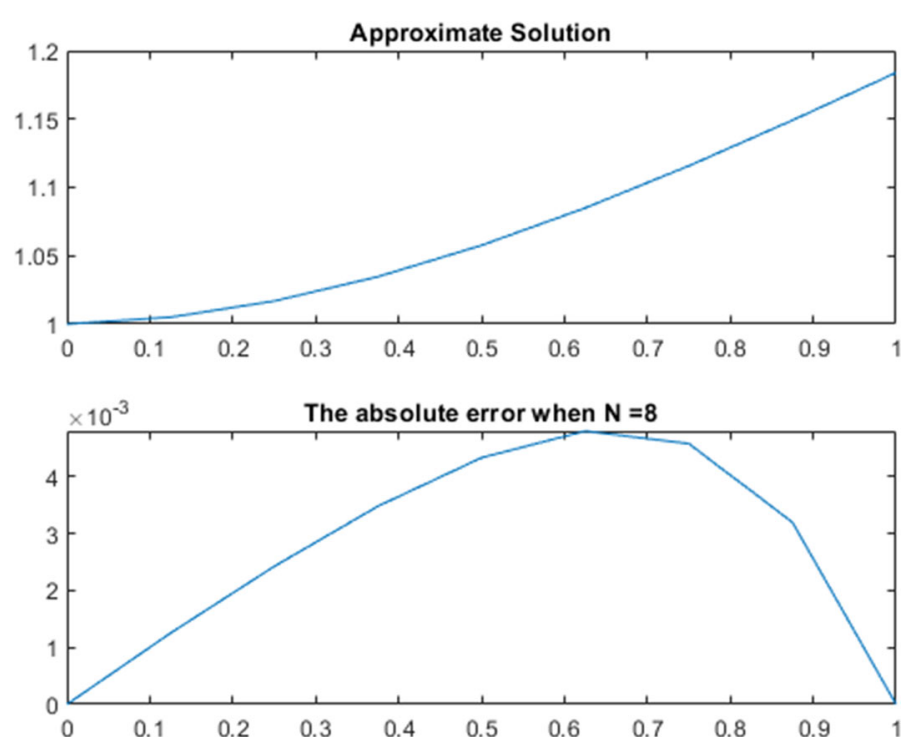

Fig. 1 The Absolute Error of test Example (1) with $N=8$.
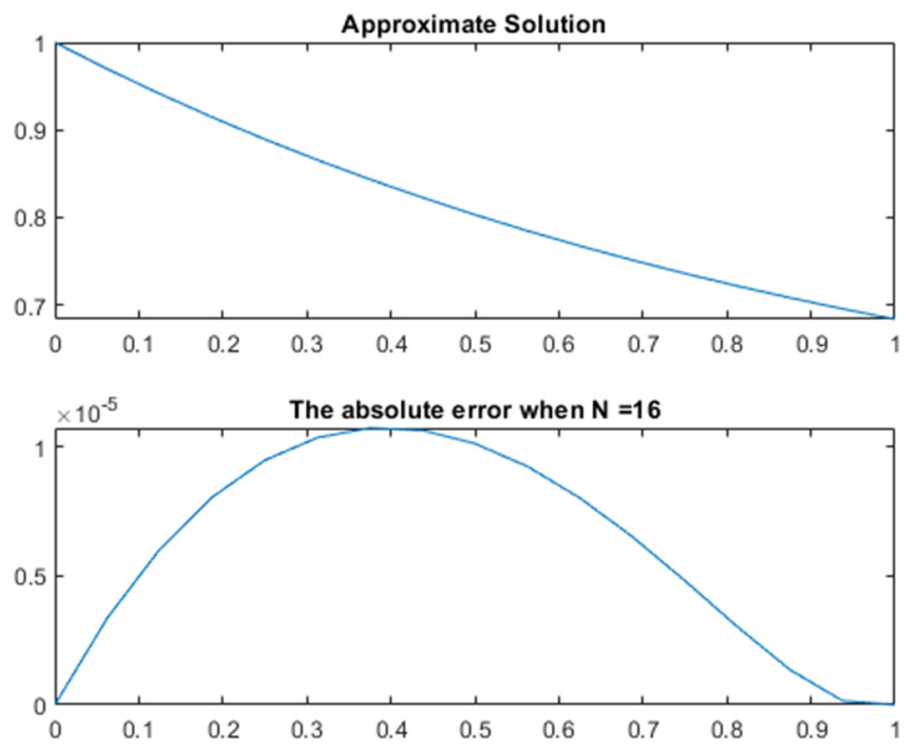

Fig. 2 The Absolute Error of test Example (2) with $N=16$.

where $\rho=1.5, \quad q(t)=1-\frac{t^{10}}{10000}, \quad f(t)=t e^{-t}-\left(\frac{t^{10}}{10000}-1\right)\left(\frac{e^{-t}-1}{2}\right)$ with the exact solution $v(t)=\frac{e^{-t}+1}{2}$. 


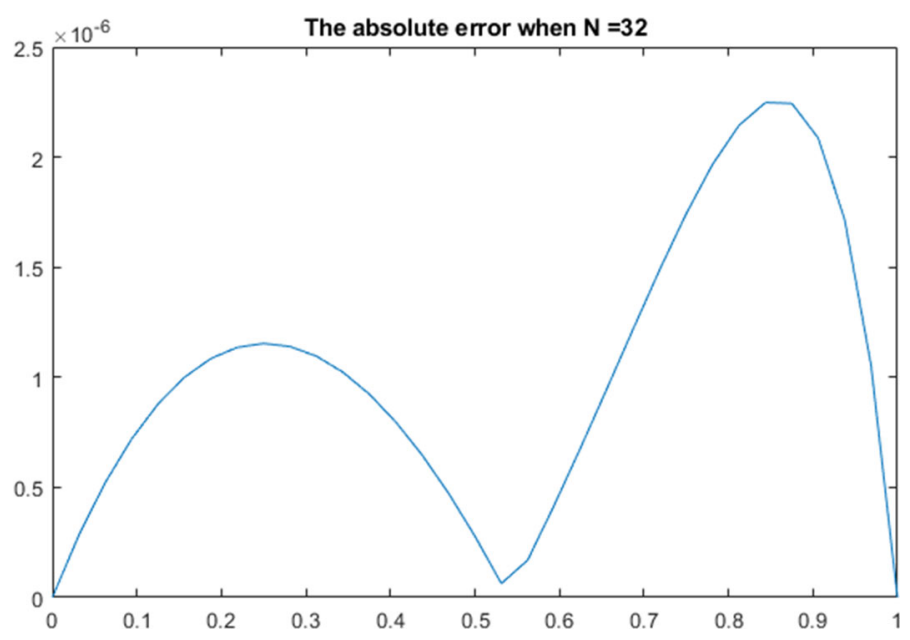

Fig. 3 The Absolute Error of test Example (2) with $N=32$.

Example 3 Consider the following fractional boundary value problem:

$$
\begin{aligned}
& \mathscr{D}^{(\rho)} v(t)+q(x) v(t)=f(t), \quad 0 \leq t \leq 1 \\
& v(0)=1, \quad v(1)=2
\end{aligned}
$$

where $\rho=1.75, q(t)=(1-t) / 2$, and $f(t)=-100 t e^{2 t}$. In this case, we don't know the exact solution.

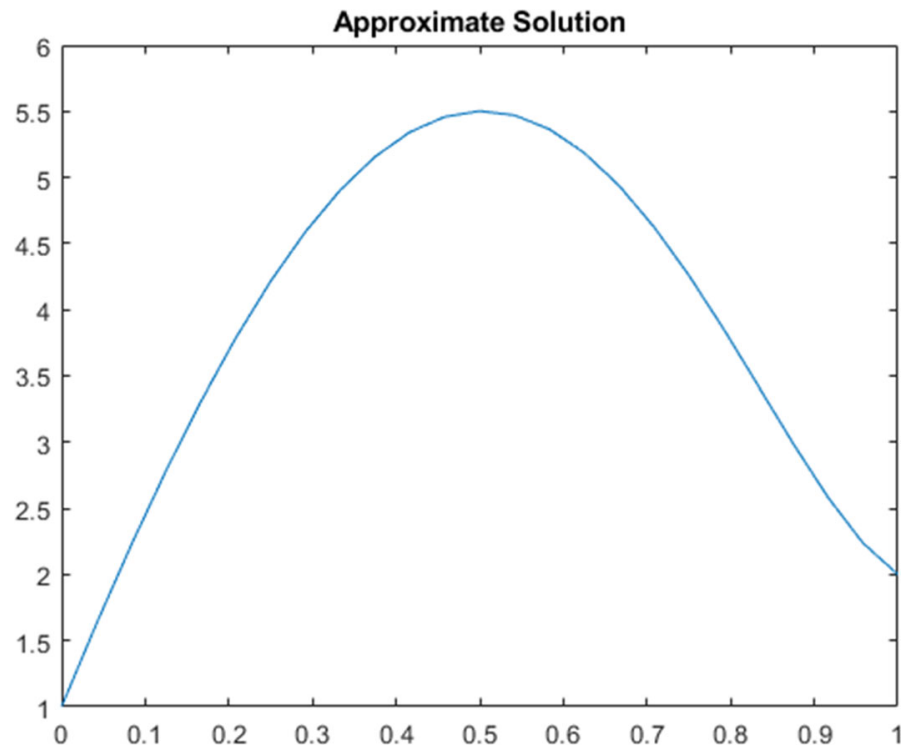




\section{Conclusion}

In this article, we proved the existence and uniqueness of the fractional boundary value problem with use of the minimum of hypotheses that ensure this, and using numerical methods and programming by Matlab to solve the problem.

\section{References}

1. Atanagana, A., Baleanu, D.: Caputo-Fabrizio derivative applied to groundwater flow within confined aquifer. J. Eng. Mech. 143(5), D4016005 (2017). https://doi.org/10.1061/(ASCE)EM.1943-7889. 0001091

2. Atangana, A., Gómez-Aguilar, J.F.: A new derivative with normal distribution kernel: theory, methods and applications. Physica A 476, 1-14 (2017)

3. Atangana, A., Gómez-Aguilar, J.F.: Numerical approximation of Riemann-Liouville definition of fractional derivative: from Riemann-Liouville to Atangana-Baleanu. Numer. Methods Partial Differ. Equ. 34, 1502-1523 (2018)

4. Aydogan, S.M., Baleanu, D., Mohammadi, H., Rezapour, S.: On the mathematical model of Rabies by using the fractional Caputo-Fabrizio derivative. Adv. Differ. Equ. 2020, 382 (2020)

5. Balenu, D., Jajarmi, A., Mohammadi, H., Rezapour, S.: A new study on the mathematical modelling of human liver with Caputo-Fabrizio fractional derivative. Chaos, Solitons and Fractals 134, 109705 (2020)

6. Balenu, D., Mohammadi, H., Rezapour, S.: A fractional differential equation model for the COVID-19 transmission by using the Caputo-Fabrizio. Adv. Differ. Equ. 2020, 299 (2020)

7. Bashiri T., Vaezpour S.M., Nieto J.J.: Approximating solution of Fabrizio-Caputo Volterra's model for population growth in a closed system by homotopy analysis method. J. Funct. Spaces 2018, Article ID 3152502 (2018)

8. Caputo, M., Fabrizio, M.: A new definition of fractional derivative without singular kernel. Prog. Fract. Differ. Appl. 1(2), 73-85 (2015)

9. Dokuyucu, M.A., Celik, E., Bulut, H., Baskonus, H.M.: Cancer treatment model with the CaputoFabrizio fractional derivative. Eur. Phys. J. Plus 133(92), 1-6 (2018)

10. Furati, K.M., Kassim, M.D., Tatar, N.T.: Existence and uniqueness for a problem involving Hilfer fractional derivative. Comput. Math. Appl. 64, 1616-1626 (2012)

11. Gómez-Aguilar, J.F., Atangana, A.: New insight in fractional differentiation: power, exponential decay and Mittag-Leffler laws and applications. Eur. Phys. J. Plus 132(13), 1-21 (2017)

12. Gómez-Aguilar, J.F., Yépez-Martínez, H., Calderón-Ramón, C., Cruz-Orduña, I., Escobar-Jiménez, R.F., Olivares-Peregrino, V.H.: Modeling of a mass-spring-damper system by fractional derivatives with and without a singular kernel. Entropy 17(9), 6289-6303 (2015)

13. Gómez-Aguilar, J.F., Yépez-Martínez, H., Torres-Jiménez, J., Córdova-Fraga, T., Escobar-Jiménez, R.F., Olivares-Peregrino, V.H.: Homotopy perturbation transform method for nonlinear differential equations involving to fractional operator with exponential kernel. Adv. Differ. Equ. 2017, 68 (2017)

14. Hilfer, R.: Applications of Fractional Calculus in Physics. World Scientific, Singapore (2000)

15. Linz, P.: Analytical and numerical methods for Volterra equations, vol. 7. Siam, Delhi (1985)

16. Losada, J., Nieto, J.J.: Properties of a new fractional derivative without singular kernel. Prog. Fract. Differ. Appl. 1(2), 87-92 (2015)

17. Magin, R.L.: Fractional calculus models of complex dynamics in biological tissues. Comput. Math. Appl. 59, 1586-1593 (2010)

18. Mehandiratta, V., Mehra, M., Leugering, G.: Optimal control problems driven by time-fractional diffusion equations on metric graphs: optimality system and finite difference approximation. SIAM J. Control Optim. 59, 4216-4242 (2021). https://doi.org/10.1137/20M1340332

19. Mehandiratta, V., Mehra, M., Leugering, G.: An approach based on Haar wavelet for the approximation of fractional calculus with application to initial and boundary value problems. Math. Methods Appl. Sci. 44, 3195-3213 (2021). https://doi.org/10.1002/mma.6800 
20. Mehandiratta, V., Mehra, M.: Existence results and stability analysis for a nonlinear fractional boundary value problem on a circular ring with an attached edge: A study of fractional calculus on metric graph. Netw. Heterog. Media 16, 155-185 (2021). https://doi.org/10.3934/nhm.2021003

21. Moumen, Bekkouche M., Guebbai, H.: Analytical and Numerical Study for an Fractional Boundary Value Problem with conformable fractional derivative of Caputo and its Fractional Integral. J. Appl. Math. Comput. Mech, JAMCM (2020)

22. Moumen Bekkouche, M., Guebbai, H., Kurulay, M.: Analytical and numerical study of a nonlinear Volterra integro-differential equations with conformable fractional derivation of Caputo. Ann. Univ. Craiova Math. Comput. Sci. Ser. (2020)

23. Moumen Bekkouche, M., Guebbai, H., Kurulay, M.: On the solvability fractional of a boundary value problem with new fractional integral. J. Appl. Math. Comput. (2020). https://doi.org/10.1007/s12190020-01368-x

24. MoumenBekkouche, M., Guebbai, H., Kurulay, M., Benmahmoud, S.: A new fractional integral associated with the Caputo-Fabrizio fractional derivative. Rendiconti del Circolo Matematico di Palermo Series (2020). https://doi.org/10.1007/s12215-020-00557-8

25. Oldham, K.B.: Fractional differential equations in electro chemistry. Adv. Eng. Softw. 41, 9-12 (2010)

26. Podlubny, I.: Fractional Differential Equations: An Introduction to Fractional Derivatives, Fractional Differential Equations, to Methods of Their Solution and Some of Their Applications. Academic Press, New York (1999)

27. Shaikh, A., Tassaddiq, A., Nisar, K.S., Baleanu, D.: Analysis of differential equations involving CaputoFabrizio fractional operator and its applications to reaction-diffusion equations. Adv. Differ. Equ. 2019, 178 (2019). https://doi.org/10.1186/s13662-019-2115-3

28. Veeresha, P., Prakasha, D.G., Baskonus, H.M.: New numerical surfaces to the mathematical model of cancer chemotherapy effect in Caputo fractional derivatives. Chaos 29, 013119 (2019)

29. Wazwaz, A.M.: A First Course in Integral Equations. World Scientific Publishing Company, Singapore (2015)

30. Wazwaz, A.M.: Linear and Nonlinear Integral Equations. Springer, Berlin (2011)

31. Xiao-Jun, X.J., Srivastava, H.M., Machado, J.T.: A new fractional derivative without singular kernel. Therm. Sci. 20(2), 753-756 (2016)

Publisher's Note Springer Nature remains neutral with regard to jurisdictional claims in published maps and institutional affiliations. 